\title{
Desarrollo de las estadísticas del medio ambiente: planteamientos y conclusiones ${ }^{\mathrm{a}}$
}

\author{
Alfonso Mercado, ${ }^{\mathrm{b}}$ Carlos Roberto López, ${ }^{\mathrm{c}}$ Arturo Flores, ${ }^{\mathrm{d}}$ \\ Francisco Giner, ${ }^{\mathrm{e}}$ Boris Graizbord, ${ }^{\mathrm{f}}$ Alejandro Lorea ${ }^{\mathrm{g}}$ \\ y Claudia Schatán ${ }^{\mathrm{h}}$
}

\section{Introducción}

Este simposio es resultado de la colaboración de seis instituciones y diversos expertos de reconocido liderazgo en la concepción, generación y uso de las estadísticas ambientales. Dichas instituciones representan a los sectores público, académico, internacional y privado: Dirección de Integración de Estadísticas de Medio Ambiente y Desarrollo Sustentable del Instituto Nacional de Estadística y Geografía (INEGI), Programa de Estudios Avanzados en Desarrollo Sustentable y Medio Ambiente (LEAD México) y Programa sobre Ciencia, Tecnología y Desarrollo (Procientec) de El Colegio de México, Dirección General de Estadística e Información Ambiental de la Secretaría de Medio Ambiente y Recursos Naturales (Semarnat), Sede Subregional de la Comisión Económica para América Latina y el Caribe (CEPAL) en México y Comisión de Estudios del Sector Privado para el Desarrollo Sustentable (Cespedes).

El simposio tuvo su origen en los trabajos en torno a un proyecto de mayor alcance para las estadísticas ambientales y fundamentalmente del Sistema Nacional de Información Ambiental y de Recursos Naturales y del Subsistema Nacional de Información Geográfica y del Medio Ambiente (este último como parte del Sistema Nacional de Información Estadística y Geográfica): el diseño e instrumentación de la Encuesta Nacional de Gasto y Gestión Ambiental en los Estableci-

\footnotetext{
${ }^{\text {a }}$ Relatoría del simposio "Desarrollo de las estadísticas del medio ambiente: fuentes, alcances y usos", celebrado en El Colegio de México, el 20 y 21 de octubre de 2008.

${ }^{b}$ El Colegio de México. Correo electrónico: amercado@colmex.mx.

${ }^{c}$ INEGI. Correo electrónico: robertp.lopez@inegi.org.mx.

d Semarnat. Correo electrónico: afloresm@semarnat.gob.mx.

e Grupo México. Correo electrónico: francisco.giner@mm.gmexico.com.

${ }^{\mathrm{f}}$ El Colegio de México. Correo electrónico: graiz@colmex.mx.

g Cespedes. Correo electrónico: alorea@cce.org.mx.

h CEPAL. Correo electrónico: Claudia.SCHATAN@cepal.org.
} 
mientos. Este proyecto surgió durante la Conferencia Estadística de las Américas de la CEPAL (Santiago de Chile, 2005), en la que se resaltó la importancia de que los países cuenten con fuentes periódicas de información para apoyar la toma de decisiones en materia de control y prevención de la contaminación ambiental.

Desde que se puso en marcha esta iniciativa en México se consideró necesario conjuntar esfuerzos en diversos frentes del quehacer institucional y con una perspectiva integral para el mejor desarrollo de la información: la participación de los productores y los usuarios de las estadísticas ambientales. El simposio reunió a más de 30 instituciones, cuyos representantes dictaron tres conferencias magistrales, presentaron 28 ponencias e hicieron dos presentaciones de libros. En un balance sintético, se concluye que se lograron los siguientes objetivos generales:

- Identificar las necesidades de información ambiental y analizar los avances y rezagos en materia de estadísticas ambientales para proponer nuevas estrategias y mecanismos de captación de información.

- Revisar las bases conceptuales y metodológicas y los procedimientos e instrumentos de captación de estadísticas ambientales, con el fin de mejorar su cobertura y confiabilidad.

- Fortalecer, desde la óptica del desarrollo sustentable, los sistemas de información que apoyen el diseño e instrumentación de las políticas de los sectores público y privado.

- Analizar la propuesta de encuesta sobre gasto y gestión ambiental de los establecimientos y contrastarla a la luz de las experiencias internacionales.

Teniendo como referencia la agenda y los objetivos para cada sesión, en este documento se reseñan los principales planteamientos y propuestas de los ponentes, así como las conclusiones y algunos comentarios que surgieron de la discusión. Para un análisis más amplio sobre el tema, los interesados pueden consultar la documentación siguiente: las memorias de ponencias que se presentaron en el simposio, la agenda de la actividad, los objetivos generales y específicos por mesa, y la lista de participantes. 


\section{Conferencias magistrales}

Estadísticas e indicadores ambientales en América Latina y el Caribe

En esta conferencia Jorge Máttar, de la CEPAL, parte del hecho de que América Latina es una de las regiones del mundo que pese a su mayor diversidad biológica presenta tendencias preocupantes en cuanto a su sustentabilidad ambiental: alta dependencia de las materias primas e hidrocarburos, pérdida de la biodiversidad, urbanización extensiva, alto nivel de degradación del agua, sobreexplotación de los recursos naturales y extrema vulnerabilidad ante los desastres.

El reconocimiento de esta problemática ha llevado en la última década a un avance de las estadísticas ambientales: una mayor "institucionalización" en torno a éstas, la creación de ministerios de medio ambiente, la instrumentación de programas de medio ambiente y una creciente cooperación dentro de cada país, regional y global. Estas medidas implican mayores necesidades de información ambiental, lo cual se ha traducido en desarrollos importantes en tres vertientes de la información ambiental: estadísticas, indicadores y cuentas, en las temáticas convencionales: biodiversidad, suelos, recursos hídricos, océanos, costas y mares, atmósfera, desastres naturales, patrones de producción y consumo sustentables, transporte, asentamientos humanos y gestión ambiental.

Todos los avances se han reflejado en publicaciones e informes sobre estadísticas, indicadores y cuentas ambientales. Entre las estrategias para impulsar el desarrollo de la información, destacan las siguientes tendencias:

- Hay una creciente identificación de los problemas ambientales desde una dinámica global que lleva a una agenda internacional.

- El fenómeno de la transversalidad del medio ambiente debe acarrear mayores esfuerzos de coordinación y cooperación.

- Es importante apoyar las iniciativas para el mejoramiento de las estadísticas ambientales, especialmente en su armonización para facilitar su comparabilidad. 


\section{Hacia el Subsistema Nacional de Información Geográfica y del Medio Ambiente en el marco de la SNIEG}

Enrique de Alba Guerra, del INEGI, explicó en esta conferencia que deben tenerse en mente dos aspectos fundamentales en el camino a la consolidación del Subsistema Nacional de Información Geográfica y de Medio Ambiente (SNIGMA): a) los retos de organización y coordinación que impone el marco constitucional y legal de la nueva Ley del Sistema Nacional de Información Estadística y Geográfica (SNIEG) y, b) los avances trascendentales en materia de proyectos, productos e información ambiental, en sus dos componentes: geográfico y del medio ambiente.

En cuanto al primer aspecto, debe considerarse que en México el Estado contará con un SNIEG, es decir, un conjunto de unidades productoras de información, organizadas en tres subsistemas y articuladas mediante la Red Nacional de Información para producir y difundir la que sea de interés nacional, que tendrá carácter oficial. Esta información está integrada por los censos, los índices de precios y las cuentas nacionales, además de la que satisfaga los cuatro criterios siguientes: a) trate los temas aludidos por la Ley, $b$ ) sea necesaria para sustentar el diseño y la evaluación de las políticas públicas de alcance nacional, c) sea generada en forma regular y periódica, y $d$ ) se elabore con base en una metodología científicamente sustentada. Asimismo la que sirva para prevenir y atender emergencias o catástrofes originadas por desastres naturales y la que atienda compromisos establecidos en algún tratado internacional.

Las unidades que conforman el sistema tienen atribuciones para desarrollar actividades estadísticas y geográficas y mantener registros administrativos que permitan obtener información de interés nacional. Dicho sistema se compone de tres subsistemas: sociodemográfico, económico, y geográfico y del medio ambiente, así como de otros agrupamientos que pueden ser temáticos, transversales o administrativos.

Los siguientes órganos colegiados conforman la estructura del SNIEG en cuatro niveles: $1^{\circ}$ ) La Junta de Gobierno y Consejo Consultivo Nacional, que coordinan el sistema, $2^{\circ}$ ) Los comités ejecutivos, que dirigen a los subsistemas, $3^{\circ}$ ) Los comités técnicos especializados (temáticos y transversales) y el coordinador de unidades.

En cuanto al segundo aspecto, se han logrado notables avances en proyectos, productos e información ambiental. En el componente geográfico, el SNIGMA ha logrado los siguientes desarrollos de infor- 
mación ambiental: por un lado, la carta topográfica, que constituye la base sobre la cual se construyen "edificios lógicos" de información ambiental, y por otro, el inventario de información de los recursos naturales, con coberturas nacionales en diversos temas y en diferentes escalas, que alimenta la base de datos geoespaciales.

En lo que se refiere al componente ambiental, y en relación con el quehacer de la Semarnat, el SNIGMA cuenta con información ambiental sobre temas prioritarios nacionales: Indicadores Básicos del Desempeño Ambiental de México, Sistema Nacional de Indicadores Ambientales, publicaciones de Estadísticas e Informes del Medio Ambiente, así como la Base de Datos Interactivos; todos ellos alimentan al Sistema Nacional de Información Ambiental y de Recursos Naturales. Por el lado del INEGI, el SNIGMA dispone de los siguientes avances: compendios ambientales nacionales (en colaboración con la Semarnat), compendios ambientales de las zonas metropolitanas (en colaboración con los gobiernos estatales), indicadores que atienden prioridades nacionales con la coordinación de organismos internacionales (desarrollo sustentable, de seguimiento, transporte en América del Norte, energía sustentable).

Con base en lo anterior se presentaron los desafíos fundamentales para la producción e integración de la información del SNIGMA: realizar periódicamente diagnósticos de la información disponible, atender las necesidades de los usuarios en las estrategias de desarrollo de dicha información, considerar la infraestructura humana y técnica de las instituciones y actuar de manera coordinada para la consolidación del SNIEG.

\section{Cambio climático: necesidades de información}

Para dimensionar la problemática del cambio climático, Mauricio Alarcón, de la Semarnat, planteó la necesidad de observar el comportamiento de la concentración global del $\mathrm{CO}_{2}$ atmosférico. Refirió que aumentó de 280 partículas por millón ( $\mathrm{ppm}$ ) que tenía a principios de la revolución industrial $(\approx 1750)$ a 385 ppm en la actualidad, el valor más alto de los últimos 650 mil años y probablemente de los últimos 20 millones de años. Tan sólo en el periodo comprendido entre 2000 y 2006 el crecimiento de la concentración promedio global del $\mathrm{CO}_{2}$ atmosférico fue de $1.93 \mathrm{ppm}$ al año, mientras que la tasa para los 30 años previos había sido de 1.5. 
En este contexto, en 1994 entró en vigor la Convención Marco de las Naciones Unidas sobre Cambio Climático que incluyó los términos de entendimiento de los países sobre este problema global; en 2005 se puso en marcha el Protocolo de Kioto, una especie de reglamento que busca la reducción de los gases de efecto invernadero (GEI).

Frente a las evidencias de los impactos del cambio climático en diferentes regiones del mundo, el mayor desafío de nuestra generación es lograr que hacia el año 2050 se hayan reducido las emisiones a la mitad del nivel que tenían en el 2000.

El Programa Especial de Cambio Climático requiere un cúmulo de información, en un esquema que considera como ejes fundamentales: los riesgos y fenómenos extremos, la observación del fenómeno, la información sectorial, los modelos y escenarios, la comunicación y la educación, el impacto en los ecosistemas y los impactos socioeconómicos. Cada uno de estos ejes hace necesario un gran esfuerzo de generación de la información y, por ende, de coordinación entre las instituciones que participan.

\section{Mesa 1: Cambio climático}

Se planteó la importancia de la información estadística para el registro y análisis apropiado de las amenazas e impactos del cambio climático, en especial la información sobre los gases de efecto invernadero (GEI) y se hizo un llamado a que las instituciones se involucren más en el intercambio de información.

Entre las principales líneas de acción, se requiere generar y actualizar la información y crear bases de datos en todos los sectores económicos; la creación urgente de una sola base de datos nacionales, aunque el problema es ¿quién la instrumentará?

El Instituto Nacional de Ecología (INE) suele encargarse de la modelación de los escenarios futuros en colaboración con algunas instituciones del extranjero, pero faltaría que el INEGI ordenara las estadísticas básicas y pusiera a la disposición de los interesados una sola base de datos.

Se exhortó también a que las instituciones generadoras de información trabajen conjuntamente, sobre todo en las estadísticas básicas, identificando fuentes clave e incluyendo observaciones geoestadísticas.

Se requiere más información y mayor veracidad. Son muchas las instituciones que participan en el manejo de información, una de las cuales es el INEGI. Falta información a nivel municipal. 
Ante el fenómeno del cambio climático hay en México una "adaptación silenciosa" e ineficiente, un "comportamiento defensivo" que no responde con medidas frontales de solución, sino que tiende a moverse hacia otras alternativas depredadoras o intensivas. El rendimiento agrícola está en declive por el cambio climático. Hay cambios de cultivos hacia los de menor rendimiento, aunque más resistentes. Se incrementan las áreas de cultivo a costa de reducir las de bosques y selvas y, como consecuencia, tiende a afectarse a la biodiversidad.

Se habló de la economía del cambio climático en México y de los escenarios que involucra en materia de consumo de energía y agua, así como de bosques, para lo cual es indispensable contar con información relacionada con la evolución y las tendencias del desarrollo tecnológico, con los patrones de consumo, y con el crecimiento de la población, entre otras cuestiones.

El reto central es hacerse de instrumentos de medición muy sólidos. Los procesos de estandarización y una adecuada red de divulgación son fundamentales para construir una infraestructura de datos espaciales accesibles.

Se exhortó a diseñar "círculos virtuosos", con un enfoque holístico y sistémico de geografía y medio ambiente. Es necesario brindar una atención especial a los desastres naturales, con datos recabados antes del desastre y después del mismo.

Respecto al cambio climático es preciso: $a$ ) identificar cuáles son las variables que hay que medir y b) reconocer que todo fenómeno ambiental tiene una referencia en el espacio.

Frente a esta problemática se planteó la propuesta de organizar la información conforme a una perspectiva integral, no sólo referente al cambio climático.

La propuesta es que los datos deben georreferenciarse con visión holística y sistémica y que puedan compartirse en una red de conocimiento. Esta propuesta aterriza en la Infraestructura de Datos Espaciales de México (Idemex) del componente geográfico del SNIGMA.

Respecto al inventario de emisiones de gases de efecto invernadero se plantearon tres puntos críticos:

a) Hay retrasos en la expedición de los datos.

b) Los indicadores no los elabora el INEGI sino el INE.

c) No se cuenta con una compilación estadística comparable en el tiempo; solamente hay estudios de casos. 
Se sugirió que el INEGI elabore el inventario. Un problema al respecto es que dicho inventario se basa en reportes voluntarios; se sugiere que la Cédula de Operación Anual (COA), que alimenta al Registro de Emisiones y Transferencia de Contaminantes (RETC), sea obligatoria y por planta. También se observa que hay grandes huecos de información en varios sectores; por ejemplo, no hay información sobre transporte marítimo.

\section{Comentarios generales}

Conviene aprovechar más la información estadística sobre cambio climático de la que ya se dispone. Se sugirió impulsar los programas voluntarios para reducir las emisiones de gases de efecto invernadero. Hay que crear más información selectivamente y en coordinación con los usuarios de la misma. Se sugirió difundir información por zonas o regiones en el interior de cada estado. Asimismo aplicar encuestas de seguimiento regional y cobertura de los sectores.

\section{Mesa 2: Energía}

\section{Relevancia de las estadísticas de energía}

La relevancia de las estadísticas de energía se manifiesta en los siguientes cuatro aspectos: $a$ ) la eficiencia energética es crucial para el cambio climático, pues según el World Energy Outlook de 2007, 40\% de la reducción de emisiones está relacionado con la eficiencia energética; b) los agentes de todos los sectores requieren información sobre energía para tomar decisiones, por lo que, aunque sea costoso construir información, no tenerla lo es más; c) la energía no es un bien homogéneo (commodity), que se pueda intercambiar sin problemas en el mercado, sino un bien medular para la actividad económica, y $d$ ) la información sobre energía es crucial para definir las políticas públicas y hacer su seguimiento.

\section{Problemática sobre las estadisticas de energía}

Es complejo elaborar indicadores de eficiencia energética, pues se requiere información muy detallada sobre los patrones de consumo 
de una gran cantidad de fuentes. De esto surgen dos problemas primordiales: 1) la generación de la información y 2) la homogeneización de las definiciones (no sólo sobre unidades de medición, también sobre tipo de combustible). Como el grado de resolución de la información es muy importante, ésta puede diferir si se recolecta a nivel macro o micro.

Los problemas específicos alrededor de las estadísticas energéticas son:

a) El proceso de desregulación del sector ha multiplicado las fuentes.

b) La confidencialidad impide compartir información.

c) Esto genera una mayor carga de trabajo en las oficinas de estadística, pues ahora deben atender más empresas y en el futuro inmediato sectores emergentes, como en el caso de las fuentes renovables.

d) Los recursos disponibles para la producción de estadísticas no corresponden a la carga de trabajo.

e) Es alta la rotación de los recursos humanos en estas instituciones, lo que reduce su experiencia.

f) Existe una gran falta de cooperación entre las oficinas de estadística y las de planeación.

g) Las empresas tienen que satisfacer varios requerimientos y existe duplicidad de información (tanto la Sener como la Semarnat aplican encuestas de uso de energía); hay además proliferación de conceptos sin definición homogénea.

Se hizo un recuento de problemas según las fuentes estadísticas. La información de la Semarnat para el sector energético está muy agregada, lo que reduce su utilidad. El Inventario Nacional de Emisiones (elaborado por el INE) muestra también algunos problemas (como se comentó en la sesión de Cambio Climático), sobre todo los supuestos de homogeneidad entre las fuentes generadoras. Pemex produce información muy detallada sobre descargas, consumo de agua, generación de residuos, uso de energía y gasto en inversión ambiental, pero es limitada en el tiempo. Esta empresa ha hecho un gran esfuerzo por consolidar la información, pero al agregarse, los usuarios difícilmente pueden localizarla, pues la encuentran sólo parcialmente. Aunque hay mucha información, no se presenta de forma accesible al público. Tanto el Instituto de Investigaciones Eléctricas como el Instituto de 
Investigaciones Nucleares generan información sobre la infraestructura de fuentes renovables y alternas, así como sobre la inversión en investigación y desarrollo y recursos humanos.

\section{Necesidades de información}

Las necesidades de estadísticas energéticas evolucionan junto con los cambios en el ambiente económico. Por ejemplo, durante el shock petrolero de los años setenta se requería información sobre los niveles de producción y las reservas, y actualmente hay necesidad de información sobre emisiones. Se mencionaron dos grandes retos en cuanto a nueva información. Primero, para dar respuesta al cambio climático se requiere información detallada. Segundo, es necesario también calcular la huella de la energía por producto, no sólo para calcular el impacto ambiental de la demanda final, también para mapear los flujos de energía y de materiales a través del comercio internacional.

La información sobre energía en México está muy fragmentada. Es necesario ampliarla y mejorarla para: integrar los aspectos financieros relacionados con la utilización de fuentes renovables, mejorar y ampliar los datos sobre eficiencia energética, cuantificar los distintos impactos ambientales del uso de la energía, incluir información sobre investigación y desarrollo, así como analizar los efectos de la aplicación de los instrumentos regulatorios. Esto es particularmente importante en el contexto de la nueva Ley de Fuentes Renovables de Energía. También existe un gran vacío en términos de información regional.

La información del sector energético para atender la problemática del cambio climático en sus procesos de adaptación y mitigación, así como el inventario de emisiones de GEI es insuficiente. Según el perfil energético de México, convendría mejorar primordialmente el potencial de energías renovables y el potencial de eficiencia energética. El impuesto al carbono y otros mecanismos económicos requieren, entre otras acciones, establecer una línea de base, lo que exige información muy detallada.

El sector transporte presenta problemas específicos. Se mencionó que $80 \%$ de las empresas del sector autotransportes no entrega datos a la Comisión Nacional para el Ahorro de Energía (convertida recientemente a Comisión Nacional para el Uso Eficiente de la Energía, Conuee) a pesar de los obvios beneficios de mantener esa contabilidad. Los empresarios atienden más a los tiempos de operación que a los 
indicadores de eficiencia energética. Es necesario, por lo tanto, un cambio en la cultura empresarial, pero también en la cultura del consumo, y es preciso difundir información sobre la eficiencia energética de los bienes de uso final, como los automóviles.

Finalmente se destacó que en el país se padecen problemas de subconsumo de energía o pobreza energética, ya que los pobres a menudo carecen de acceso a fuentes energéticas asequibles, fiables, seguras y ambientalmente benignas, lo que afecta seriamente sus condiciones de vida. En otras palabras, conviene conocer la proporción de sus ingresos que dedica cada familia al consumo de combustibles. Habría que generar este indicador en el futuro.

Se mencionó que si se incluyera en los Censos Económicos 2009 un conjunto de preguntas sobre el tipo y volumen de los combustibles que utilizan los mayores consumidores se satisfarían algunas de las necesidades de información.

\section{Recomendaciones}

Para afrontar la problemática de las estadísticas de energía, se formulan las siguientes 10 recomendaciones:

a) Lleva tiempo construir sistemas de información confiables; se han logrado avances, pero falta mucho por hacer.

b) Hay que orientar la información a la necesidad del usuario. Se necesita repensar el paradigma de la generación de datos. Hay que entender qué información requiere el usuario y cuál es la información útil, no simplemente producirla desde las instituciones. Pemex, por ejemplo, ha elaborado una iniciativa de participación ciudadana para guiarse sobre la necesidad de información de los usuarios.

c) Es muy importante que la información necesaria para atender el cambio climático responda a las especificidades del país y no sólo a los lineamientos internacionales. Es crucial definir qué tipo de información es más relevante.

d) Dar importancia a la medición de las necesidades cotidianas, es decir, la que sirve a todos los individuos para tomar decisiones (estadísticas de eficiencia en consumo final).

e) Buscar en lo posible que la información sea validada por terceros. 
f) Son indispensables la armonización de conceptos y la cooperación entre instituciones: hay que establecer bases normativas y marcos de referencia para evitar repetición de esfuerzos. Es necesario unificar los objetivos, las definiciones y los criterios, así como coordinar las acciones de las diversas instituciones para facilitar su cooperación y mejorar la calidad de la información.

g) Evitar la duplicación de datos y producir el dato una sola vez para que se use muchas veces.

h) El INEGI debería desempeñar una labor más intensa: por un lado, precisar la manera en que se integra la información y cuáles son los mejores procedimientos para procesar los datos y, por otro, definir qué información necesita el país, desde los tomadores de decisiones, los investigadores, etc. Esto es particularmente urgente en el caso de la información necesaria para responder al cambio climático.

i) La nueva Ley del SNIEG facilita el camino para resolver muchos de estos problemas, pero es necesario transitar por él. La Ley misma es una guía para llevar a cabo tres programas de desarrollo de la información: anual, sexenal (que incluye cuestiones de diseño y tratamiento de información), y estratégico (a 24 años). Estos programas deberían alimentarse con las necesidades de los usuarios para construir una mejor estadística. Finalmente, la Ley es un buen marco para el INEGI, pero se requiere voluntad política para aplicarla; si no, será letra muerta. Con eso deben comprometerse las autoridades a muchos niveles.

\section{Mesa 3: Agua}

\section{Situación presente}

Actualmente se cuenta con información sobre extracción anual, disponibilidad promedio nacional, cobro por derechos de agua, tanto proveniente de fuentes oficiales como de la academia y ONG o consultorías. Se dispone de datos del agua superficial y subterránea en distintas escalas cartográficas, así como bases de datos que posibilitan su manejo ágil e integrado. El monitoreo que realizan varias instituciones ha llevado al desarrollo de ciertos sistemas como el de observación climatológica de la Conagua y el Sistema de Aguas de la Ciudad de 
México. Las cuentas económicas y ecológicas también requieren información, que al procesarse resume (en unidades físicas y monetarias) la situación actual de los recursos hídricos. Adicionalmente la OCDE, en su revisión del desempeño ambiental de México y con base en la información disponible, hace recomendaciones al país sobre la gestión del agua. Los censos y conteos de población y vivienda ofrecen información sobre el acceso al agua y al drenaje, lo mismo que los censos económicos respecto a los organismos operadores.

\section{Perspectivas}

Se requiere integrar la información conforme a los flujos medio ambiente-economía propuestos por la ONU, mediante el sistema de recursos hídricos y el sistema económico y de los usos del agua; asimismo se requiere producir información acerca de la calidad del agua y en escalas a mayor detalle que las actuales. Por otro lado conviene eliminar las diferencias entre los datos y las dificultades para acceder y disponer de ellas. De manera complementaria habrá que proponer algunos indicadores relacionados con el agua. Conforme a las recomendaciones de la OCDE, se requiere desarrollar instrumentos estadísticos para dimensionar adecuadamente la importancia del recurso, evaluar los flujos de origen y destino, y contar con un balance de las existencias, entre otros aspectos.

Ha sido muy importante la cooperación interinstitucional para el desarrollo y mejora de la información hidrológica y estadística, aunque deberán hacerse esfuerzos para mejorar su calidad, teniendo en cuenta que no es posible contar con indicadores confiables sin la estadística básica suficiente.

Es necesario considerar la nueva información acerca de modificaciones en el uso del agua, su producción y el consumo directo o indirecto, su calidad estructural como agua muerta o viva, lo mismo que el ahorro que conlleva el empleo de nuevas tecnologías; corregir diferencias de conceptos, mejorar el acceso y disponibilidad de los datos en internet, así como superar con el trabajo conjunto los huecos de información, sobre todo respecto a los datos primarios y a la operación de redes de acceso. 


\section{Mesa 4: Residuos}

\section{Problemática}

Uno de los grandes problemas de la información ambiental es la incertidumbre en las cifras de los residuos, pues es desigual y no está sistematizada. En México las cifras relativas a los residuos sólidos urbanos (de jurisdicción municipal) son convenidas, inferidas y obsoletas, y provienen además de distintas instituciones. Es preciso mejorar tal información considerando los siguientes tres hechos: a) ninguna entidad federativa cuenta con un inventario detallado de la cantidad y composición de los residuos, como tampoco sobre la eficiencia ni la efectividad de la infraestructura, $b$ ) sus inventarios se concentran en la disposición final, y c) no hay datos suficientes sobre los costos variables (tarifas) de la administración de los residuos sólidos urbanos.

Respecto a los residuos de manejo especial (de jurisdicción estatal), hay poca información y la disponible está dispersa; no se cuenta con inventarios; hay incertidumbre en su definición y caracterización, falta metodología y se carece de planes para su manejo.

Por otro lado, sobre los residuos peligrosos (de jurisdicción federal) se cuenta con un inventario parcial, y los manifiestos e informes que entregan los generadores presentan muchas deficiencias en sus datos.

\section{Sugerencias}

Se precisa actualizar y homologar las metodologías, la clasificación de los residuos y su composición, y mejorar las proyecciones. El INEGI puede aportar experiencias para homologar las metodologías. Dada la relevancia del tema que nos ocupa, sería importante crear un grupo temático dentro de los comités del SNIEG. En la Ley General para la Prevención y Gestión Integral de los Residuos se establece que habrá de crearse un sistema de información relativa a la generación y gestión integral de los residuos sólidos urbanos y los residuos de manejo especial, cuyo objetivo será desarrollar inventarios con información actualizada sobre los indicadores de desempeño de la gestión integral de residuos.

Para lograr lo anterior es preciso definir el modelo conceptual de Gestión Integral de Residuos y los criterios para la recolección de los 
datos, así como identificar las fuentes de información y las herramientas con que se levantará la información (un cuestionario general y otro complementario que contendrán varios anexos referidos a la generación, la composición y otros temas). Se espera que el sistema esté operando para el año 2012.

En lo que se refiere a los residuos peligrosos, la nueva legislación del reglamento de la ley define una serie de instrumentos de gestión para sistematizar y explotar de las fuentes de información para la gestión integral de este tipo de residuos.

Su aplicación favorecerá grandes avances, como los siguientes: a) contar con información veraz y oportuna, $b$ ) obtener una visión integral de la problemática sobre los residuos peligrosos, c) ser una herramienta de información para estructurar las políticas públicas, $d$ ) desarrollar líneas de investigación científica para el manejo integral, e) promover el desarrollo de infraestructura para el manejo integral municipal y estatal, f) facilitar el acceso público a la información sobre el tema (transparencia), $g$ ) establecer convenios.

A pesar de las limitaciones de la información sobre los residuos, se ilustraron con un caso los usos posibles que pueden hacerse de la información disponible; se trató del estudio "Indicadores sobre el desempeño ambiental industrial en México”, referido a la emisión de contaminantes relacionados con el valor y el volumen de producción de las actividades económicas, incluso por entidad federativa.

\section{Mesa 5: Desarrollo tecnológico y sustentabilidad}

\section{El gasto en investigación y desarrollo para la sustentabilidad ambiental}

Si el gasto federal en ciencia y tecnología es bajo, el gasto en investigación dedicado al medio ambiente lo es todavía más. El gasto federal en ciencia y tecnología representa apenas $0.37 \%$ del PIB y de éste sólo $1.7 \%$ se destina al medio ambiente y los recursos naturales.

En el subíndice Manejo Sustentable y Medio Ambiente del Instituto Mexicano para la Competitividad (Imco), México ocupa el último lugar en el contexto de América Latina, y el costo hipotético en inversión es de 770 dólares por trabajador (estimado a partir del promedio de los 10 países que ocupan los primeros lugares en el subíndice). Entre los principales retos destacan: el tratamiento de agua, la calidad de los combustibles, y el reciclaje. En materia de información se re- 
quiere una desagregación estatal relativa a la sobreexplotación de los cuerpos de agua, la deforestación anual y el monitoreo de los programas de reforestación, entre otros.

\section{Financiamiento de la investigación y el desarrollo proambientales}

De la inversión nacional en ciencia y tecnología, $46.7 \%$ lo financia el gobierno, $44.4 \%$ el sector productivo y las familias, $7.7 \%$ las instituciones de educación superior y $1.2 \%$ fondos del exterior. Se cuenta con el Fondo Sectorial de Investigación Ambiental Semarnat-Conacyt como programa de apoyo gubernamental de proyectos que cuentan con "méritos tecnológicos" en investigación y desarrollo para que se les seleccione.

\section{Estadísticas disponibles}

El aprovechamiento de las encuestas que levanta el INEGI junto con otras dependencias permite perfilar algunas estadísticas sobre medio ambiente; sin embargo es necesario construir un sistema de encuestas o módulos. Actualmente el INEGI, en coordinación con el Conacyt, lleva a cabo la Encuesta sobre la Investigación y Desarrollo Tecnológico (Esidet) y el Módulo de Innovación Tecnológica (MIT). La Esidet tiene por objeto medir los recursos humanos y financieros dedicados a actividades de investigación y desarrollo en los sectores productivos, de educación superior, de gobierno y en las instituciones privadas no lucrativas. El MIT permite conocer la importancia del objetivo de innovación para desarrollar productos que no afecten al medio ambiente o reduzcan el consumo de energía.

Es recomendable, con un esfuerzo adicional mínimo, aprovechar las preguntas que ya formulan el cuestionario de la Encuesta y el MIT e introducir nuevas opciones. Algunos ejemplos: $a$ ) gasto en servicios de consultoría en materia ambiental, $b$ ) pago por asistencia técnica, consultorías y trabajos de ingeniería para instrumentar tecnologías limpias, c) inversión estimada para transformar los procesos de producción a un esquema de industria limpia, $d$ ) frecuencia con que la empresa renueva o modifica sus procesos para incorporar tecnologías limpias.

Otra estrategia para obtener estadísticas de medio ambiente y tecnología, sobre todo cuando la información proviene de registros 
administrativos o de censos, es utilizar a los diversos clasificadores con un mayor nivel de desagregación. Por ejemplo, podría utilizarse la Nomenclatura Internacional Normalizada de la UNESCO relativa a campos y disciplinas de la ciencia.

\section{Comentarios y sugerencias}

Se reconoce el avance logrado en cuanto a las estadísticas de medio ambiente y tecnología; sin embargo éstas no se utilizan por desconocimiento o desconfianza. Hay ausencia de investigación y de estadísticas del cambio tecnológico pro ambiental. Para la protección ambiental no sólo se requiere el desarrollo de más investigación en la materia, además se debe considerar la adquisición de tecnología. Hay asimismo muchos cambios tecnológicos con fines de lucro que tienen consecuencias favorables para el medio ambiente.

La carencia de estadísticas confiables se debe quizá a que la temática es relativamente nueva. Hay un exceso de confianza al suponer que las tecnologías y la innovación son la solución a todos los problemas, pero, por el contrario, más bien son parte del problema. Más que nuevos desarrollos científicos y tecnológicos se requiere saber utilizar y aprovechar las tecnologías correctamente de modo que causen el menor daño posible, ya que se sabe que algunas provocan destrucción.

\section{Mesa 6: Hogares y medio ambiente}

Se revisaron las experiencias nacionales e internacionales en la recolección de estadísticas sobre las prácticas de los hogares respecto del cuidado ambiental y el uso de los recursos naturales, y se propusieron algunos instrumentos de captación necesarios para obtener dicha información.

\section{El cuidado ambiental en los hogares}

La participación de los hogares y sus miembros en actividades de conservación y aprovechamiento ocurre en varios ámbitos: el hogar, la familia, la comunidad y el gobierno. Por ejemplo, es importante iden- 
tificar las reglas que siguen las comunidades para definir quiénes pueden participar en las actividades de conservación y aprovechamiento, así como conocer el tipo de tenencia de la tierra, las jerarquías de toma de decisiones y sus relaciones en el hogar, la familia, la comunidad y el gobierno, entre otras. Es muy importante conocer cómo inciden las características demográficas del hogar (sexo, edad, parentesco) en la posibilidad de que sus miembros participen en actividades de conservación del medio ambiente. También interesan los conocimientos de sus integrantes sobre el potencial que ofrece la biodiversidad local para el desarrollo de su familia y su comunidad, y si hacen uso de ellos, además de otros factores como sus actitudes, su participación en la toma de decisiones y en la organización de actividades de aprovechamiento, y su interés en la conservación del medio.

Los hogares desempeñan un doble papel: el de consumidores de recursos naturales y el de productores de emisiones de gases, residuos domésticos y aguas residuales. Las investigaciones han destacado la importancia de su dinámica en el acceso, uso y control de los recursos naturales. La reducción del tamaño de los hogares promueve un uso menos eficiente de los recursos de las viviendas que se podría considerar una deseconomía. Por otro lado, si bien el ritmo de crecimiento poblacional ha disminuido, quienes estén en edades casaderas requerirán más viviendas, lo que incrementará la demanda de suelo urbano, el abastecimiento de servicios de agua, luz, pavimento y transporte, y acarreará un impacto ambiental importante. Urge, entonces, contar con estadísticas ambientales provenientes de censos y encuestas de hogares sobre el acceso, uso y control de los recursos.

Recientemente comenzó a investigarse el cuidado ambiental en los hogares de México, no sólo en los urbanos, también en los rurales, y entre éstos en los de áreas campesino-indígenas, como los de Chihuahua y Yucatán con un clima extremo. Dos indicadores cualitativos se consideran relevantes: a) los estilos de vida, en el que se engloban las migraciones temporales por la baja en la producción agrícola y la falta de agua entubada, que obliga a recurrir a la captación de agua de las laderas de los cerros por medio de tuberías de plástico; y b) las creencias, dentro del cual se considera la exposición de la población a las ideas occidentales sobre el cuidado de la salud y el medio ambiente.

Para satisfacer sus necesidades de información cualitativa y cuantitativa los académicos han realizado trabajo de campo directo enfocado en particular en los estilos de vida, el uso de los ecosistemas, el grado de urbanización, el acceso a sistemas de agua y drenaje, el siste- 
ma cultural que regula la interacción con el medio ambiente y las percepciones de riesgo.

En cuestiones forestales, por ejemplo, hay gran interés por dos temas: a) la influencia de la estructura institucional del ejido en la incorporación de las actividades de aprovechamiento y conservación forestal a las estrategias familiares, y b) la comparación del tipo de estrategias familiares desarrolladas por los hogares, con el interés de analizar la importancia relativa del recurso forestal dentro de esas estrategias.

\section{Una encuesta levantada por la OCDE}

La OCDE concluyó recientemente una encuesta sobre el comportamiento ambiental en los hogares que abarca cinco áreas clave: los desechos, la energía, el agua, la comida orgánica y el transporte. Se aplicó a través de internet a una muestra de 10000 hogares en 10 países: Canadá, Noruega, Francia, Italia, Corea del Sur, México, Suecia, Holanda, Australia y República Checa.

De acuerdo con esta encuesta, en México el tiempo de desplazamiento promedio en el transporte público es mayor que en otros países, se ubica en el último lugar de estos 10 países en el uso de energía renovable, registra niveles bajos en el consumo de comida orgánica y su reutilización del agua es inferior a la de los demás. Sin embargo, México muestra una elevada preocupación por la contaminación del aire y por el cambio climático, y su inquietud por el uso de bolsas recicladas es muy parecida a la del resto de los países.

En la encuesta también se encontró que la escolaridad y el conocimiento ambiental son variables relevantes para acceder a un mundo limpio, por lo cual se invita al INEGI a que incluya estos aspectos en las estadísticas que genera.

\section{Comentarios y sugerencias}

No existe información en México sobre la magnitud del impacto ambiental de los hogares. Ante esta realidad la Encuesta Nacional de Ingresos y Gastos en los Hogares (ENIGH) será una fuente de información muy valiosa para estudiar las tendencias del gasto ambiental de los hogares y para hacer inferencias sobre el consumo nacional, dado que la encuesta de 2008 tendrá representación estadística en las 32 
entidades federativas. Es necesario captar y difundir más información estadística al respecto; afortunadamente se está planeando realizar en el año 2010 una encuesta sobre el consumo y el comportamiento ambiental de los hogares urbanos y rurales.

También hay gran preocupación por el consumo de biomasa en México, especialmente en relación con los hogares rurales. Es necesario saber cómo interactúan los hogares con el consumo de biomasa y cómo y cuánto invierten las entidades federativas en la difusión y en la educación ambiental sobre este tema. Dada la gran escasez de estadísticas al respecto, se sugiere maximizar su difusión. También se requiere una eficiente interacción del INEGI y la academia.

\section{Mesa 7: Empresa y medio ambiente}

\section{La medición del gasto ambiental en América Latina}

La respuesta de los sectores público y privado a los problemas ambientales puede abordarse desde dos perspectivas: por los cambios producidos en los recursos y por el gasto ambiental que realizan esos sectores. Debe considerarse que en el caso de las empresas el gasto en protección ambiental está ligado a la productividad. Por gasto ambiental se entiende lo invertido en las actividades para la protección del medio, lo cual incluye: prevención, reducción y eliminación de contaminantes en la producción y consumo de bienes. Se debe incluir por lo tanto el gasto realizado por las empresas y los hogares.

Hay diversos criterios respecto a la clasificación del gasto en protección ambiental: puede ser gasto de inversión o corriente, de capital, o como transferencia que proviene del gobierno; otra opción es atender a su origen: del gobierno, de las empresas o de los hogares. La CEPAL propone usar la Clasificación de Actividades y Gasto para la Protección Ambiental (CEPA-CAPA 2000, por sus siglas en inglés) que permite la comparación internacional, pues está consensuada por la ONU, la OCDE y Eurostat. Estudios hechos por la CEPAL y el PNUMA en algunos países de América Latina muestran que el gasto público como porcentaje del PIB que se invierte en gasto ambiental va de 0.3 a $0.6 \%$, mientras que en el resto del mundo se ubica en alrededor de $1 \%$. En los países desarrollados además del gasto público destinado a la protección ambiental se invierten recursos del sector privado, que van de 0.2 a $0.9 \%$ del PIB; en México la cifra se ubica en 0.4 por ciento. 
En resumen: el gasto en protección ambiental no supera $1 \%$ del PIB y, el del sector público rara vez pasa de $3 \%$ del total. Es preciso considerar los problemas que acarrea el captar la información, como los gastos que hacen las empresas en rubros no considerados ambientales y que indirectamente benefician al medio y no se contabilizan en el rubro correspondiente.

\section{El gasto privado en protección ambiental}

¿Por qué los países requieren la información que generan las empresas respecto a su propio gasto ambiental?, ¿por qué las empresas deben gastar en ello y en qué les beneficia? y ¿por qué les conviene informar sobre sus gastos ambientales? Para responder estos cuestionamientos un estudio de la CEPAL partió de tres condicionantes: a) que las empresas estaban dispuestas a gastar en la protección ambiental, b) que los gobiernos precisaban esta información para elaborar políticas públicas, y c) que la sociedad civil y sus organizaciones desempeñaran un papel ambiental más activo.

Un primer desafío para captar la información ambiental es la elaboración y aplicación de encuestas específicas en la materia, a pesar de las limitaciones propias de la utilización de cuestionarios, que por ejemplo captan información cuantitativa y no cualitativa. Un segundo desafío está en una clara definición del concepto "gasto ambiental", dado que comprende más elementos que la protección ambiental. Finalmente, un tercer desafío es que las oficinas de estadística definan los sectores que deben considerarse para aplicar las encuestas.

En Canadá se cuenta con cinco encuestas: gasto en protección ambiental, industrias ambientales, gestión de desechos para los sectores privado y público, hogares y medio ambiente. La información que se genera suelen utilizarla frecuentemente los usuarios debido a que se trata de encuestas hechas a petición de ellos mismos. La información que se obtiene se utiliza para formular indicadores de la calidad del agua, la calidad del aire y las emisiones de gases de efecto invernadero.

La CEPAL realizó en América Latina un ejercicio sobre la factibilidad de las encuestas en materia de gasto ambiental; sus resultados no fueron satisfactorios debido, entre otras causas, a la insuficiente información ambiental que se genera en los sectores público y privado, a la saturación de encuestas, a que pocas empresas tienen un sistema con- 
table eficiente, y al escepticismo sobre la confidencialidad de la información. En Brasil las encuestas privadas son buenas, pero su información se refiere a aspectos muy puntuales y por lo tanto poco relevantes para las políticas públicas. En Colombia predomina una situación parecida a la de Brasil, a lo que debe agregarse que los cuestionarios son diferentes cada vez que se diseñan, lo cual impide comparar los datos. Finalmente, en Chile las encuestas son buenas con un nivel de respuesta de 90 por ciento.

\section{¿Qué medimos en realidad como protección ambiental?}

La protección ambiental no puede ser vista como algo lineal, ya que el peso de los gastos de las empresas es muy variable. Por ejemplo, en un auto nuevo que no necesita inversiones para no contaminar, éstas ya están incorporadas en el costo de la unidad; otro caso es el de un auto construido unos años atrás que requiere una inversión regular para mejorar su eficiencia y no contaminar; y un tercer caso es el de un auto viejo que requiere un gasto fuerte para mejorar su eficiencia, el cual puede ser igual al costo de la unidad. La pregunta para los tres casos es: ¿quién invierte más en protección ambiental, el que más gasta o el que es más eficiente?

Las encuestas no miden el gasto en protección sino en control de la contaminación, lo cual lleva a una percepción equivocada de las empresas. Hoy día las encuestas solamente captan las acciones que se realizan al final del proceso productivo y no durante éste. Además, el control no resuelve los problemas de la contaminación, sino la introducción de nuevas tecnologías y el mejoramiento de los equipos. Muchas veces las inversiones para mejorar los procesos son superiores a la exigencia ambiental.

Se debe tener cuidado con los datos del desempeño ambiental, pues esa información muestra los resultados al final del proceso y no los del proceso de prevención y, por tanto, no revela cuánto se contribuye a mejorar el ambiente, sino a no ensuciarlo.

Encuesta del INEGI sobre gasto y gestión ambiental

El propósito de la Encuesta Nacional de Gasto y Gestión Ambiental (ENGGA), a cargo del INEGI, es generar estadísticas e indicadores sobre 
los gastos y la gestión ambiental de los establecimientos del sector industrial en las ramas de manufactura, minería, servicios y transporte. Los impactos ambientales de estas ramas son fuertes. La importancia de la encuesta deriva de los esfuerzos de coordinación que se han hecho entre algunas instituciones de la academia, de la iniciativa privada y del sector público. Asimismo, de las propuestas de nuevas variables de carácter ambiental introducidas en el Censo Económico 2009 surgirán resultados de interés para la estadística ambiental. Se ha hecho una prueba piloto para evaluar la formulación de las preguntas y se está en una fase de ajustes para una segunda prueba.

Respecto a la prueba piloto, que se aplicó entre las ramas de transportes de residuos peligrosos, fabricación de envases de cartón, rastros, vidrio, equipo agrícola, laboratorios, talleres mecánicos, entre otras, el nivel de respuesta fue alto (90\%); la mayoría de los entrevistados pidió que se le dejara el cuestionario para llenarlo posteriormente argumentando que requería la asesoría de instancias contables. En las pequeñas y medianas empresas participó más de un área en el llenado. Los informantes de la encuesta fueron muy diversos: desde gerentes hasta jefes de ecología, de seguridad o varios a la vez.

En cuanto a la información, su validación fue difícil y sólo $50 \%$ cumplió con los requisitos que la hicieran satisfactoria. En la parte cualitativa de la información no se presentaron problemas con los grandes y medianos establecimientos, pero sí con las pequeñas empresas; por ejemplo, no conocen la normatividad y por lo tanto mantienen sus patrones de contaminación, como en los talleres mecánicos, que siguen tirando el aceite sucio al drenaje público. Por otra parte se encontró que en algunas microempresas, como los laboratorios químicos, el control de los registros es confiable respecto al manejo de sus residuos.

El reto fundamental es crear un sistema de encuestas ambientales en temas complementarios, para lo cual se requerirá la colaboración de diversas instituciones.

\section{Las encuestas ambientales en Estados Unidos}

Las encuestas ambientales en Estados Unidos, que incluían el costo y el gasto en protección ambiental, se venían haciendo desde 1973. En 1994 el gobierno decidió cancelarlas, pero se renovaron en 1998 como respuesta a las necesidades de información de la Agencia de Protección 
Ambiental (EPA) sobre los gastos en este rubro. Las encuestas fueron pagadas por esa institución hasta 2005, cuando se decidió reestructurarlas y no continuaron más. Era obligatorio que las contestara la industria. Los datos revelan que en 2005 el sector manufacturero erogó 21 billones de dólares en gastos en protección ambiental, lo cual representó $0.5 \%$ de lo invertido en ese sector.

Si bien las encuestas están dirigidas a captar la inversión al final del proceso, también proporcionan información sobre la inversión en los procesos productivos, como la compra de equipo para prevención. No se incluyen los gastos que no están dirigidos específicamente a la protección del ambiente.

Los cambios en las encuestas ambientales en Estados Unidos han modificado su estructura integrando una visión más amplia del concepto de gasto ambiental, para lo cual colaboraron ingenieros, economistas y otros profesionistas. Se realizaron pruebas piloto que, aunadas a los conocimientos de los expertos, mejoraron las encuestas, ya que tras más de tres años de funcionamiento era necesario renovarlas.

\section{Comentarios}

Aún no hay información suficiente para saber si las empresas cumplen con la normatividad ambiental y si ha mejorado su eficiencia, en particular sus ciclos de crecimiento. La información oficial es poca y muy dispersa, por lo que subsisten grandes retos en materia de desempeño y protección ambiental.

La Profepa propone compartir experiencias con el INEGI y Cespedes para construir indicadores de desempeño ambiental.

El gran reto es cambiar las sanciones por los incentivos para que las empresas dejen de considerar un castigo o una carga la entrega de información.

Como resultado de las exposiciones sobre el tema "Empresa y medio ambiente" se propuso convocar a los participantes a una reunión de trabajo para evaluar la posibilidad de revisar el estado actual de los Indicadores de Desempeño Ambiental. 


\section{Mesa 8: Hacia un sistema integrado de estadísticas del medio ambiente}

\section{La disponibilidad de la información ambiental en México}

De acuerdo con el marco de indicadores de la OCDE México sólo cumple con la elaboración de dos tercios de la información ambiental. Además de tal rezago, la brecha entre la disponibilidad y las necesidades de información al respecto ha venido ampliándose. Esto se observa especialmente en el transporte y la energía.

Es notable la dispersión institucional en la generación de información, por lo que se requiere trabajar dentro de un marco conceptual para unificar criterios y estándares. El INEGI habrá de afrontar este problema desempeñando su papel como institución coordinadora de los esfuerzos para la elaboración de esta información.

Es urgente avanzar en la interrelación de lo científico, lo estadístico y lo geográfico (por ejemplo respecto al cambio climático). Hay que fortalecer la base estadística de información ambiental e incorporar la de los censos, las encuestas y los registros administrativos.

Se sugiere captar información en los tres elementos del desarrollo sustentable: población, economía y medio ambiente, conforme a las categorías sugeridas por la ONU.

Los sistemas de estadísticas ambientales deben vincularse con los de cuentas ambientales y los de información geográfica siguiendo las recomendaciones internacionales de vinculación de la información. También es importante mejorar la coordinación entre las unidades productoras de estadísticas ambientales. Habrá que profundizar las interrelaciones de causas y efectos buscando la integración de esfuerzos y la coordinación de las fuentes primarias.

\section{El sistema de información ambiental en México}

¿En qué consiste la interacción del Sistema de Información Ambiental de la Semarnat con el subsistema del INEGI? ¿Cómo van a integrarse a la luz de la promulgación de la Ley del INEGI?

Es importante encontrar las áreas de convergencia para llenar los huecos de información del Sistema Nacional de Información Ambiental y de Recursos Naturales. ¿Cuál es la información que realmente se debe incorporar? ¿Son los hogares, las empresas, el cambio climático, 
el agua, los suelos o las cuestiones económicas?, ¿con qué desagregación y detalle: local, estatal, nacional?

Otra tarea es evaluar las relaciones costo-beneficio de la disposición de información que haya sido generada. ¿Sirve y se explota? La información digital que menos se consulta es la estadística. El objetivo será que se genere información y además se utilice.

La confiabilidad de la información también es crucial. La falta de homologación entre diferentes fuentes contribuye a deteriorarla, por lo que es urgente homologar las bases de datos de las dependencias gubernamentales. Este reto resulta mayúsculo.

Es vital avanzar hacia el sistema integrado de estadísticas del medio ambiente. Es prioritario atender la realidad de la interacción de proveedor y usuario en un sistema integrado de estadísticas ambientales. Debe darse una vinculación interinstitucional clara que satisfaga la demanda y escale en un aprendizaje continuo con la retroalimentación de los usuarios. Frente a estos desafíos es necesario generar un sistema de calidad. Por todo ello se recomienda consolidar esfuerzos para no generar información escasa y dispersa y avanzar de manera sistemática.

Entre los elementos base para transitar hacia un sistema de información estadística destaca la claridad de los acuerdos interinstitucionales, para que garanticen la colaboración y se logre una asignación específica de recursos. Una herramienta fundamental es el uso de clasificaciones claras, consistentes y homogéneas, así como metodologías comunes.

El objetivo final es lograr la generación de "un solo dato", y la estrategia consiste en contar con una base de datos común y captar periódica y secuencialmente la información. En el caso mexicano, las bases existentes facilitarán el establecimiento del nuevo sistema que se propone.

\section{Generación de estadísticas ambientales}

La autonomía del INEGI es un hito, pues éste cuenta ahora con la facultad de normar y coordinar el sistema de información ambiental nacional. En cuanto al componente de medio ambiente del SNIGMA, tales atribuciones significan desplegar, conjuntamente con las unidades del sector ambiental, las actividades necesarias para el desarrollo de las estadísticas ambientales. El rol del INEGI tendrá que allegar recursos a esta tarea. 
Se prevén los siguientes pasos:

a) La Junta de Gobierno del INEGI definirá las características, el contenido y los alcances del Subsistema.

b) Se crearán nuevas instancias de coordinación del Subsistema, como los Comités Ejecutivos y los Comités Técnicos Especializados. La Ley faculta al INEGI para ser coordinador entre niveles de gobierno.

c) Establecer los criterios de planeación, impulsando el programa estratégico, el plan nacional y después un plan específico.

d) Decidir las prioridades dentro de los requerimientos de ampliación de la información.

e) Actualizar y precisar las normas técnicas.

La Ley ya define información que se considera de interés nacional, como los censos, los índices de precios y las cuentas nacionales. La información que se genere deberá corresponder a las determinaciones de la Ley.

Para las cuestiones ambientales emergentes se requerirá el desarrollo de más estadísticas ambientales. Esta información debe ser utilizable; debe fungir como base para tomar decisiones consistentes. En suma, la generación de información constituye un reto para fortalecer las alianzas entre las dependencias generadoras.

\section{La experiencia en Estados Unidos}

En Estados Unidos ya se comenzaron a revisar los procesos de la contabilidad ambiental. Esta experiencia lleva a recomendar que se integre la contabilidad ambiental y que se tome en cuenta la información sobre energías renovables y otras similares sin valor de mercado, como la del agua potable y la del aire puro. En la contabilidad ambiental estadounidense destacan los siguientes tres desafíos:

a) La contabilidad ambiental no está reconocida legalmente como requisito y no hay fondos destinados a tal objetivo. Por ello hasta hace poco en Estados Unidos no se contaba con fondos para financiar su continuidad.

b) Hay problemas sobre la escasa disponibilidad, la confiabilidad y la calidad de los datos. 
c) La contabilidad del sector público en Estados Unidos se está rezagando respecto a los demás sectores productivos. El sector académico está analizando las tendencias del daño y el efecto ambientales de todas las actividades económicas.

Al hablar de la generación de la estadística es importante el acceso público a la información. En especial interesa la información de la actividad industrial sobre aspectos como el consumo de energía y el tipo de energéticos utilizados (se detectaron cerca de 20 tipos de energéticos en el sector industrial). Es relevante también la carencia de series de tiempo confiables, y es preciso transitar hacia la integración de información relevante entre las dependencias. El verdadero avance en la captación y análisis de información ambiental en Estados Unidos proviene fundamentalmente de la academia.

Hacia un sistema integrado de estadísticas del medio ambiente en México

- Se requiere la caracterización puntual de los parámetros ambientales que conviene medir y que éstos realmente puedan ser cuantificados.

- Las estadísticas deberán generarse de manera sistemática, periódica y con calidad, sin descuidar lo ya logrado y sin perder de vista la coordinación y vinculación entre las unidades generadoras.

- Toda la información que se elabore dentro del marco que otorga la Ley SNIEG deberá disponerse para su uso efectivo. 


\section{ANEXO}

\section{Agenda del simposio "Desarrollo de las estadísticas del medio} ambiente: fuentes, alcances y usos" (octubre de 2008)

\section{Lunes 20}

Presidium: Enrique de Alba, INEGI; Jorge Máttar, Cepal; Arturo Flores, Semarnat; Alejandro Lorea, Cespedes, y Manuel Ordorica, El Colegio de México

Inauguración: Manuel Ordorica, El Colegio de México, y Enrique de Alba, INEGI

\section{Conferencias magistrales:}

- "Estadísticas ambientales en América Latina y el Caribe". Jorge Máttar, CEPAL-México

- "Políticas y estrategias de México frente al cambio climático". Mauricio Alarcón, Semarnat

- "Hacia el Subsistema Nacional de Información Geográfica y de Medio Ambiente en el marco de la LSNIEG". Enrique de Alba Guerra, INEGI

Moderador: Jaime Sempere, El Colegio de México

Mesa 1: Cambio climático

Ponentes: Antonio Mediavilla, INE; Luis Miguel Galindo, UnAM; Mario A. Reyes, INEGI, y Rodolfo Lacy, Centro Mario Molina

Comentaristas: Claudia Schatán, CEPAL-México; Francisco Takaki, INEGI, y Boris Graizbord, El Colegio de México

Moderador: Boris Graizbord, El Colegio de México

Relator: Alfonso Mercado, El Colegio de México

Mesa 2: Energía

Ponentes: Jorge Nuño, SENER; Gerardo Leyva, INEGI, y Mauricio Ugalde, El Colegio de México

Comentaristas: Carlos de Régules, Pemex; F. Javier García, Conae, y Roberto López, INEGI

Moderador: Vicente Ugalde, El Colegio de México

Relator: Francisco Aguayo, El Colegio de México 
Mesa 3: Agua

Ponentes: Ricardo Martínez, Conagua; María Perevochtchikova, El Colegio de México, y Raúl Figueroa, INEGI.

Comentaristas: Matthew Hammil, Cepal; Ismael Aguilar, ITESM; Francisco Jiménez, INEGI, y Hortencia Medina, INEGI.

Moderador: Antonio Yúnez, El Colegio de México.

Relator: Servando Aguilar, INEGI.

Mesa 4: Residuos

Ponentes: Alfonso Flores, Semarnat; Óscar Fernández, El Colegio de México; Víctor Gutiérrez, INE, y César Chávez, Semarnat.

Comentaristas: Zeferino Godínez, Sedesol, y Miguel del Avellano, INEGI Moderador: Arturo Flores, Semarnat.

Relator: Héctor Young, INEGI.

Mesa 5: Desarrollo tecnológico y sustentabilidad

Ponentes: Wilfredo Urueta, Conacyt; Manuel Molano, Imeco, y Gerardo López, INEGI.

Comentaristas: Alfonso Mercado, El Colegio de México; Francisco Aguayo, El Colegio de México, y Salvador Padilla, UMSNH, Morelia.

Moderadora: Norma P. Muñoz, CIIEMAD-IPN.

Relatora: Georgina Argáiz, INEGI.

Presentación de libros

Jenkins, R. y A. Mercado, Ambiente e industria en México, México, El Colegio de México, 2008.

Moderador: José A. Romero, El Colegio de México.

Coordinador del libro: Alfonso Mercado, El Colegio de México.

Comentaristas: Alejandro Lorea, Cespedes, y Miguel del Avellano, INEGI.

Semarnat, ¿Y el medio ambiente?: problemas en México y el mundo, México, Semarnat, 2008.

Moderador: Sergio Puente, El Colegio de México.

Coordinador del libro: Arturo Flores, Semarnat.

Comentaristas: Leticia Ruiz, INEGI, y Teresa Elizabeth Cueva, Colef.

Martes 21

Mesa 6: Hogares y medio ambiente

Ponentes: Alejandro Guevara, UIA; Mauricio Cervantes, El Colegio de México; Haydea Izazola, UAM-X; Teresa Elizabeth Cueva, Colef, y Hugo García, El Colegio de México. 
Comentarista: Hortencia Medina, INEGI, y Hugo García, El Colegio de México.

Moderadora: Bertha Helena de Buen, Semarnat.

Relatora: Ana María Hernández, INEGI.

Mesa 7: Empresa y medio ambiente

Ponentes: Georgina Núñez y Humberto Soto, CEPAL; Randy A. Becker, US Bureau of Census; Francisco Giner, Grupo México; Roberto López y Miguel del Avellano, INEGI.

Comentaristas: Ana Silvia Arrocha, Profepa, y Alfonso Mercado, El Colegio de México.

Moderador: Alejandro Lorea, Cespedes

Relator: Jorge Sánchez, INEGI.

Mesa 8: Hacia un sistema integrado de estadísticas

del medio ambiente

Ponentes: Roberto López, INEGI; Randy A. Becker, US Bureau of Census; Arturo Flores, Semarnat.

Comentaristas: Humberto Soto, CEPAL; Boris Graizbord, El Colegio de México; Alejandro Lorea, Cespedes, y Jesús Romo, INEGI.

Moderadora: Claudia Schatán, CEPAL.

Relator: Mauricio Ugalde, El Colegio de México.

Conclusiones: Roberto López, INEGI.

Clausura: Julie Lennox, CEPAL. 